\title{
Ziconotide Trialing by Intrathecal Bolus Injections: An Open-Label Non-Randomized Clinical Trial in Postoperative/Posttraumatic Neuropathic Pain Patients Refractory to Conventional Treatment
}

Emmanuel Bäckryd, Jan Sörensen and Björn Gerdle

Linköping University Post Print

\section{Tweet}

N.B.: When citing this work, cite the original article.

Original Publication:

Emmanuel Bäckryd, Jan Sörensen and Björn Gerdle, Ziconotide Trialing by Intrathecal Bolus Injections: An Open-Label Non-Randomized Clinical Trial in Postoperative/Posttraumatic Neuropathic Pain Patients Refractory to Conventional Treatment, 2015, Neuromodulation (Malden, Mass.), (18), 5, 404-413.

http://dx.doi.org/10.1111/ner.12293

Copyright: Wiley: 12 months

http://eu.wiley.com/WileyCDA/

Postprint available at: Linköping University Electronic Press

http://urn.kb.se/resolve?urn=urn:nbn:se:liu:diva-120352 


\section{Ziconotide trialing by intrathecal bolus injections: An open- label non-randomized clinical trial in postoperative/posttraumatic neuropathic pain patients refractory to conventional treatment}

\section{Abstract}

Objectives: The aim of this open-label, non-randomized, clinical trial was to evaluate the feasibility of trialing ziconotide by intrathecal bolus injections.

Material and Methods: Twenty-three patients, who had peripheral neuropathic pain refractory to pharmacological treatment and were under consideration for Spinal Cord Stimulation, received up to three ziconotide bolus injections according to a comprehensive algorithm. After a first injection of 2.5 $\mu \mathrm{g}$, the patients progressed in the algorithm depending on the presence or absence of pain reduction and significant adverse events. A patient was considered a "responder" if experiencing pain reduction and no significant adverse event on two consecutive occasions at the same dosage.

Results: We found a low proportion of responders (13\%). However 30\% of patients experienced $\geq 30 \%$ pain reduction on a least one injection, yielding a number needed to treat of $\sim 3$ for clinically significant pain relief. Pain intensity changed significantly over time $(0-6 \mathrm{~h})(\mathrm{p}=0.047)$ after a mean ziconotide dose of $2.75 \mu \mathrm{g}$. Adverse events were as expected, and no serious adverse event occurred. We did not find any statistical association between response to Spinal Cord Stimulation and response to ziconotide.

Conclusions: Ziconotide bolus injection trialing seems feasible, but the proportion of responders in the present study was low. Adverse events were as expected, and no serious adverse event occurred. The predictive power of ziconotide bolus trialing remains unclear, and the pharmacological profile of ziconotide (slow tissue penetration due to high hydrophilicity) calls the rationale for bolus trialing into question.

Keywords: bolus; intrathecal; spinal; trialing; ziconotide 


\section{Introduction}

Continuous intrathecal infusion of morphine in humans was first described in 1981 (1). Since then, the practice of intrathecal analgesia (ITA) has undergone a tremendous development, not least concerning infusion devices. ITA is considered to play a small but important part in the treatment of intractable cancer pain (2-4). For chronic non-cancer pain patients, however, the use of ITA is controversial (5). A systematic review from 2007 concluded that in chronic non-cancer pain patients, pain intensity seems to improve with ITA, but opioid doses increase over time and long-term effects remain unclear, due to lack of long-term follow-up (i.e. >2-3 years) (6). The recommendation strength for ITA has been described as "moderate" in cancer pain and as "limited to moderate" in non-cancer pain (7). The majority of patients treated with ITA have non-cancer types of pain (8).

The most significant ITA-related development in recent years has been the introduction of ziconotide, a synthetic analogue of a conopeptide found in the venom of the fish-hunting marine snail Conus magus (9). Ziconotide is a non-opioid that selectively blocks N-type voltage-sensitive calcium channels in the Rexed lamina I and II of the spinal cord dorsal horn $(10,11)$. The effectiveness of ziconotide has been assessed in three randomized controlled trials (12-14). It is since 2007 considered to be a first-line ITA drug $(15,16)$, ziconotide and morphine being the only analgesics approved for intrathecal infusion for pain control by the Food and Drug Administration (9). Typical opioid adverse events (AEs) such as respiratory depression, tolerance or dependence have not been described for ziconotide (17). However, ziconotide has a narrow therapeutic window $(11,17)$ and several neurological AEs have been reported, e.g. dizziness, ataxia, abnormal gait, nystagmus, or nausea. Ziconotide AEs tend to occur more commonly at higher doses (18, 19), and a slow-titration strategy is recommended (11). Due to the risk of psychiatric AEs (11, 20, 21), patients with a history of psychosis should not be treated with ziconotide $(11,22)$. Patients have to be closely monitored for cognitive impairment, hallucinations or changes in mood (19).

Before starting long-term ITA, patients often undergo an ITA-trial in order to evaluate the efficacy and tolerability of the planned treatment (23). Trial practices vary widely (24). Ziconotide trialing can be done either by continuous intrathecal infusion via an external pump, or by bolus injections (23). Bolus trialing is simpler and cheaper (25), but the evaluation is more difficult since the duration of the clinical effect is brief. Ziconotide has been administered by bolus injections in one earlier study by Mohammed et al (26) (who followed an expert-based, agreed-upon algorithm), as well as in small studies that have not hitherto been published in peer-reviewed journals (27-29).

The aim of this open-label, non-randomized, phase II clinical trial was to evaluate the feasibility of intrathecal bolus injections trialing of ziconotide (Prialt $\left.{ }^{\circledR}\right)$ in patients with posttraumatic and/or postoperative, peripheral, neuropathic pain refractory to conventional pharmacological treatment, using the same agreed-upon algorithm as Mohammed et al (26). 


\section{Materials and Methods}

\section{PATIENTS}

Patients with peripheral, postoperative/posttraumatic, neuropathic pain were invited to participate. Inclusion criteria were 1) patient, at least 18 years of age, suffering from chronic ( $\geq 6$ months) pain, who had failed on conventional pharmacological treatment; only patients with peripheral neuropathic pain due to trauma or surgery were included; 2) Visual Analogue Scale Pain Intensity during week before inclusion $\geq 40 \mathrm{~mm}$ (VASPI-inclusion); 3) patient capable of judgment, i.e. able to understand information regarding the drug, the mode of administration and evaluation of efficacy and side effects; 4) signed informed consent.

Exclusion criteria were 1) limited life expectancy (investigator's judgement); 2) intrathecal chemotherapy; 3) known or suspected intracranial hypertension; 4) known liver or kidney disease, defined as serum transaminases, total bilirubin, alkaline phosphatase or creatinine >1.2 Upper Limit of Normal (ULN); 5) advanced cardio-pulmonary disease (investigator's judgment); 6) ongoing infection, whether systemically or locally in the lumbar area; 7) coagulopathy (including medication with warfarin, clopidogrel and heparin); 8) allergy to ziconotide or any of the excipients in the ziconotide vial; 9) history of psychiatric disorders which in the investigator's opinion would put the patient at risk; 10) pregnant or lactating woman (menstruating women had to use contraceptives during the trial period); 11) participation in another clinical trial during the last 30 days.

Basic demographic data were collected. Present and past medical history was taken, including concomitant medication and previous use of analgesics. A physical examination (including neurological) was performed, and the presence of neuropathic pain was assessed (30). Forty patients were considered for eligibility, 33 were assessed, and 26 were included in the study (Figure 1).

\section{EFFICACY ASSESSMENTS}

The primary outcome variable was patient-reported VASPI. A horizontal, 0-100 mm, continuous, unmarked line was used, zero representing no pain and $100 \mathrm{~mm}$ worst pain imaginable. The patients were asked to draw a line perpendicular to the VASPI-line at the appropriate place (31). Immediately before each ziconotide injection, VASPI was reported for three time frames: past week (VASPI-7d); past $24 \mathrm{hrs}$ (VASPI-24h); current (VASPI-now). After each ziconotide injection, patients reported VASPI-now hourly for 6 hours (separate sheets of paper were used for each hourly rating), and the mean of these 6 postinjection values was calculated (VASPI-mean). Ziconotide-induced Percentage Pain Reduction (PPR) was calculated using pre-injection VASPI-now and post-injection VASPI-mean (i.e., the mean of the 6 postinjection values). At 6 hours post-injection, the Patient Global Impression of Change (PGIC-6h) was also recorded. PGIC is a 7-point scale that ranges from "very much improved" to "very much worse" with "no 
change" as the mid-point (32). At 24 hours post-injection, the patient graded VASPI-24h, VASPI-now and PGIC for the past 24 hours (PGIC-24h).

\section{SAFETY ASSESSMENTS}

Before each ziconotide injection, a blood sample for Creatine Kinase (CK) was taken (17). Moreover, a pregnancy urine test was obtained from all menstruating women. An intravenous glucose infusion was started before each injection, and the patients remained fasting for 6 hours. During the first hour postinjection, they remained in the supine position and vital signs (heart rate, blood pressure and oxygen saturation) were recorded every 15 minutes. Thereafter, vital signs were recorded hourly for 5 hours. Mean arterial pressure (MAP) was calculated using systolic blood pressure (SBP) and diastolic blood pressure $(\mathrm{DBP})$ as follows: $\mathrm{MAP}=(\mathrm{SBP}+(2 * \mathrm{DBP})) / 3(33)$. At 6 hours post-injection, the protocol stated that the patient could either stay in the hospital overnight or be discharged (investigator's judgment).

An Adverse Event (AE) was defined as any undesirable experience appearing within 24 hours after each injection, whether or not considered related to intrathecal ziconotide. All AEs reported spontaneously by the subject or observed by the investigators were recorded in a specific AE-form and in the medical record. The intensity was graded by the investigator on a 3-point scale: mild (discomfort but no disruption of normal daily activity); moderate (discomfort sufficient to reduce or affect normal daily activity); severe (inability to perform daily activity). An AE of moderate or severe intensity was considered to be significant, and only significant AEs influenced the way patients progressed in the study algorithm (see below). For each adverse event the relation to the ziconotide bolus was rated as: probable; possible; unlikely; not assessable.

In accordance with the principles of Good Clinical Practice (GCP, see below), the occurrence within 24 hours after each injection of an AE that was fatal, life-threatening, disabling or required/ prolonged inpatient hospitalization or caused congenital anomaly was defined in the protocol as a Serious Adverse Event (SAE). Furthermore, if not listed in the Summary of Product Characteristics (SPC) or in the official Swedish medicines compendium (www.fass.se), a SAE was defined as a Suspected Unexpected Serious Adverse Reaction (SUSAR), prompting immediate report to the relevant authorities.

\section{TRIALING PROCEDURE}

Commercially available ziconotide (Prialt ${ }^{\circledR}$ ) was used. Apotek Produktion \& Laboratorier (APL), one of the leading contract manufacturers in Life Science in Scandinavia (www.apl.se), diluted Prialt $®$ according to the standards of the Swedish Medical Products Agency (MPA), and vials containing $2 \mathrm{ml}$ of Prialt ${ }^{\circledR} 5$ $\mu \mathrm{g} / \mathrm{ml}$ were delivered and stored in a refrigerator at $5{ }^{\circ} \mathrm{C}$. Immediately prior to each injection, the investigator (EB) drew the appropriate volume of Prialt $5 \mu \mathrm{g} / \mathrm{ml}$ and diluted it with physiologic saline under aseptic conditions to a total volume of $2 \mathrm{ml}$. A standard lumbar puncture was performed (without fluoroscopy, preferably at level L3-L4 or L4-L5), and the appropriate dose of ziconotide was injected 
during one minute without barbotage. A 27G pencil-point (noncutting) Whitacre needle (BD Medical, Franklin Lakes, New Jersey, USA) was used. No pre-injection myelogram was performed.

Each patient received up to three ziconotide bolus injections according to a comprehensive expert-based, agreed-upon algorithm (Figure 2). The first bolus dose was always $2.5 \mu \mathrm{g}$ of ziconotide. After this first injection, the way the patient progressed in the algorithm depended on the presence or absence of pain relief (defined as PPR $\geq 30 \%$ ) and on the presence or absence of a significant AE (as defined above). Briefly, if the patient experienced:

- no pain relief and a significant $\mathrm{AE}$, no second injection was given

- no pain relief and no significant AE: the dose of the second injection was increased to $3.75 \mu \mathrm{g}$

- pain relief and no significant AE: a dose of $2.5 \mathrm{mcg}$ was repeated for confirmatory purposes

- pain relief and significant AE: the dose of the second injection was decreased to $1.25 \mu \mathrm{g}$

Following the same principles, a third injection could be given to patients who had received 1.25 or 3.75 $\mu \mathrm{g}$ at second injection. For details, see the algorithm presented in Figure 2. One week \pm three days elapsed between each injection.

A patient was considered a "responder" if experiencing pain relief (PPR $\geq 30 \%)$ and no significant AE (as defined above) on two consecutive occasions at the same dosage. All other cases were defined as "nonresponder". Moreover, PPR $\geq 30 \%$ was considered clinically significant (32).

Before or after participating in the study, some patients also tried Spinal Cord Stimulation with an externalized electrode (test-SCS).

\section{QUALITY CONTROL AND ETHICS}

Ziconotide (Prialt ${ }^{\circledR}$ ) is approved for intrathecal continuous infusions, not bolus injections. Hence, the relevant authorities in Sweden considered that the standards of ICH-GCP (International Conference of Harmonization - Good Clinical Practice) had to be applied in this study. Accordingly, this EudraCT 2010018920-21 study was conjointly approved by the MPA and the Regional Ethics Committee (REC) in Linköping, Sweden. Study monitoring was effectuated by the Linköping Academic Research Center (LARC), based at University Hospital, Linköping, Sweden. The study protocol was pre-published on-line on ClinicalTrials.gov (identifier NCT01373983), which is a registry database of publicly and privately supported clinical studies for human subjects. ClinicalTrials.gov is a service of the US National Institutes of Health.

\section{STATISTICS}


For all analyses, we used IBM® SPSS ${ }^{\circledR}$ Statistics Version 21. Unless stated otherwise, results of continuous variables are given as mean \pm SEM. As stated above in the "Trialing procedure" subsection, patients were descriptively classified as responders or non-responders according to how the followed the study algorithm (Figure 2). Because of the complicated data structure generated from the study algorithm (each patient receiving one, two or three bolus injections), a summary measures approach was chosen for inferential statistics (34). Hence, for patients who were injected twice or thrice, mean values were calculated at each time point for VASPI, pulse, blood pressure, PGIC and ziconotide dose, resulting in one set of outcome data for each patient. Repeated Measures Analysis of Variance (RM-ANOVA) and paired t-test were used to measure change over time. When the assumption of sphericity was not met for RMANOVA (i.e. when Mauchly's test $\leq 0.05$ ), Greenhouse-Geisser was chosen. Using a Bonferroni correction for post hoc analysis was judged to be far too conservative (high risk of Type II error); least significance difference was chosen instead in SPSS (equivalent to no correction). Moreover, Fisher's exact test or Wilcoxon signed ranks test were used when appropriate. A two-sided significance level of 0.05 was chosen for all analyses.

A retrospective power calculation revealed a power of $>99 \%$ to detect a $20 \mathrm{~mm}$ difference between VASPI-now and VASPI-mean for this sample size (paired-data calculation for 23 injected patients; probability of Type I error $=0.01$; observed standard deviation of difference between VASPI-now and VASPI-mean $=15 \mathrm{~mm}$ ). 


\section{Results}

A total of 36 injections were given to 23 patients (13 men and 10 women), aged $56 \pm 2$ years with a pain duration of $79 \pm 13$ months and a VASPI-inclusion of $71 \pm 3 \mathrm{~mm}$. All patients had at least probable neuropathic pain according to the criteria published by Treede et al (30), meaning that all had pain with a neuroanatomically plausible distribution (criterion 1), all had a history suggestive of a relevant lesion affecting the somatosensory system (criterion 2), and all had congruent neurological signs (criterion 3 ) and/or a relevant diagnostic test confirming the lesion (criterion 4). All patients but two had or had tried opioids; all patients but one were on or had tried amitriptyline and/or duloxetine; all patients but one were on or had tried gabapentinoids (gabapentin and/or pregabalin). Moreover, 18 patients also tried test-SCS. For further detailed characteristics of injected patients, see Table 1.

According to the study algorithm, three patients out of $23(13 \%)$ were responders, 17 (74\%) were nonresponders, and three (13\%) did not complete the algorithm subsequent to the first injection (Figure 2 and Figure 3). PPR for each injection is depicted in Figure 4. Although only 13\% were responders according to the strict criteria of the study, seven patients (30\%) still achieved $\geq 30 \%$ pain reduction on a least one injection, i.e. almost a third of patients experienced clinically meaningful pain reduction at some time during the trial (Figure 4). RM-ANOVA of pre-injection VASPI-now and hourly for six hours after injection revealed significant changes over time ( $\mathrm{p}=0.047$, Figure 5) after a mean ziconotide dose of 2.75 $\mu \mathrm{g}$. Pairwise post hoc analysis showed that the only time point which significantly differed from any other time point was the pre-injection VASPI-now; it differed significantly from time points $1 \mathrm{~h}, 2 \mathrm{~h}, 4 \mathrm{~h}$ and $5 \mathrm{~h}$. Moreover, VASPI-mean was significantly lower than pre-injection VASPI-now $(\mathrm{p}=0.019)$. However, preinjection VASPI-24h did not differ significantly from post-injection VASPI-24h ( $\mathrm{p}=0.078)$. The PGIC mode was "no change", both after 6 and 24 hours.

Eight out of the 20 patients completing the study algorithm were on concomitant gabapentinoids, but none of the three responders had concomitant gabapentinoids. There was no statistical association between concomitant treatment with gabapentinoids and response to ziconotide (Fisher's exact test, $\mathrm{p}=0.242$ ). Likewise, six patients out of 20 were on concomitant duloxetine or amitriptyline; none of these was a ziconotide responder. No statistical association was found between concomitant treatment with duloxetine/amitriptyline and response to ziconotide (Fisher's exact test, $\mathrm{p}=0.521$ ).

For patients injected at least twice, we were able to compare CK levels before and after the first injection. No statistically significant $\mathrm{CK}$ change was found on group level, CK being $3.52 \pm 0.77 \mu \mathrm{kat} / \mathrm{l}$ before injection and 3.15 $\pm 0.70 \mu \mathrm{kat} / \mathrm{l}$ one week after $(\mathrm{p}=0.286, \mathrm{n}=11)$. Four patients out of $11 \mathrm{had}$ a higher $\mathrm{CK}$ one week after injection compared to before, but only one of them had a clinically significant increase (from $3.4 \mu \mathrm{kat} / \mathrm{l}$ to $7.3 \mu \mathrm{kat} / \mathrm{l}$ ). Despite a second ziconotide injection one week after the first, the CK levels of that patient returned to normal after one more week (3.0 $\mu \mathrm{kat} / \mathrm{l})$ and stayed normal thereafter. 
Fifteen out of 23 patients experienced a total number of 33 AEs on 18 occasions. No SAE occurred. AEs occurring more than once were, in falling order of frequency: dizziness (9); tiredness (6); headache (5 none of them being a classical post dural puncture headache); nausea and/or vomiting (3); itch (2). All AEs were consistent with the SPC, and all resolved. 17 out of 33 AEs were mild, 16 were moderate, and none was severe. The relation to the ziconotide bolus was rated as probable in 24 cases; possible in 7; unlikely in 2; not assessable in one. When the relation to ziconotide was assessed to be probable or possible, the number of days to AE stop date was 1 (0-6) day (median, range).

We found statistically significant changes of both MAP and heart rate over time with RM-ANOVA ( $\mathrm{p}<0.001$ and $\mathrm{p}<0.001$, respectively) (Figure 6), but no patient experienced any clinically significant cardiovascular effect: no MAP was $<75 \mathrm{~mm} \mathrm{Hg}$ at any time point; the maximum percentage drop in MAP was $7 \%$ (-3\%-26\%) (median, range); the maximum percentage increase in MAP was 6\% (-2\%-21\%) (median, range); no heart rate value dropped under 50; heart rate exceeded 100 only once (value of 101).

After the first injection ( $n=23)$, all patients but three stayed overnight at the post-anesthesia care unit; the other three were discharged home after 6 hours (patient's wish and investigator's judgment). Concerning the subsequent injections $(n=13)$, the patients were discharged home after 6 hours in 9 out of 13 cases.

Sixteen of the patients who completed the study algorithm did also try test-SCS: 8 out of $16(50 \%)$ were non-responders to both test-SCS and ziconotide bolus injections; one out of $16(6 \%)$ responded to both treatment modalities; and seven out of 16 (44\%) responded to either test-SCS or ziconotide bolus injections. There was no statistical association between response to test-SCS and response to ziconotide bolus injection (Fisher's exact test, $\mathrm{p}=1.000$ ) 


\section{Discussion}

In this small cohort of neuropathic pain patients highly refractory to conventional pharmacological treatment, we have evaluated he feasibility of ziconotide bolus trialing according to a complex algorithm that took both PPR and moderate/severe AEs into account (Figure 2). We found a low proportion of responders (13\%) according to the strict criteria of the study. However, $30 \%$ of patients experienced $\geq 30 \%$ pain relief on a least one injection, yielding a Number Needed to Treat (NNT) of $\sim 3$ for clinically significant pain relief.

Hitherto, only one other study of this kind has been published in a peer-reviewed journal (26). In that study, with a very similar study algorithm to ours and a similar number of patients, Mohammed et al found a much higher proportion of responders (55\%). However, and in contrast to our study, Mohammed et al did not clearly specify what a "significant" AE was. When comparing the number of AEs (76 events in a total of 41 injections) reported by Mohammed et al to the low number of cases when AEs actually influenced the way patients progressed in the algorithm, one gets the impression that only very severe AEs were considered "significant". One could therefore speculate that the proportion of responders would perhaps have been lower in the Mohammed study had they used the same grading system for AEs as we did (with both moderate and severe AEs influencing the way patients progressed in the algorithm). One could also speculate that the proportion of responders would have been higher in our study had we defined only "severe" AEs as significant (as opposed to "moderate or severe"). This could be considered to be an important limitation in our study design. However, in the Mohammed study, 9 out of 11 responders received $2.5 \mu \mathrm{g}$ or less. Hence, based on the Mohammed study, increasing the dose to $3.75 \mu \mathrm{g}$ does not seem to generate a dramatically higher rate of responders. All in all, it is difficult to compare the way our respective cohorts flowed through the algorithm. This illustrates that even a seemingly "objective" flow chart is amendable to subjective interpretations (e.g. concerning what a "significant" AE really is). In the future, we think that ziconotide bolus injection trialing should rely on a much simpler algorithm, focusing on PPR and (perhaps) disregarding the lower dose of $1.25 \mu \mathrm{g}$ (which was not used in our study). One way to focus more on PPR would be to allow only severe AEs (i.e., inability to perform daily activities) to influence the way the patient progresses in such a trial algorithm.

The predictive value of being a responder in a ziconotide bolus injection trial is not known (23). Even though this can also be said of continuous intrathecal infusion trialing via an external pump, it could be argued that the latter method more accurately mimics the conditions of an implanted pump and therefore should be preferable. However, this assertion is not evidence-based. There is still a substantial lack of knowledge concerning the optimal method of ziconotide trialing (23).

AEs in our study were as expected according to the SPC. The incidence of post dural puncture headache is low when using pencil-point needles (35), and in this study no case of classical post dural puncture headache occurred. No SAE occurred, but the sample size was small, making it unlikely to detect rare 
reactions to ziconotide bolus injections. Likewise, because of the small sample size, we cannot state that CK testing is unnecessary in the bolus setting. The clinically significant rise of CK in one patient is difficult to interpret, as a new injection was followed by normalized levels after one week.

It is important to acknowledge that the starting bolus dose $(2.5 \mu \mathrm{g})$ is the dose commonly given per day when initiating ziconotide intrathecal infusion. Given that ziconotide is a highly hydrophilic molecule with very slow tissue penetration (10), and given that its half-life in the cerebrospinal fluid is well under 6 hours $(36,37)$, injecting high doses might be necessary for bolus trialing. Taking this pharmacological profile into account might be another argument for preferring continuous intrathecal infusion trialing via an external pump. Clearly, there is a need for more studies assessing the predictive values of different ziconotide trialing methods.

Ziconotide directly inhibits norepinephrine release, resulting in decrease in MAP, but this is said to be negligible when ziconotide is given intrathecally as opposed to intravenously (11). According to the SPC, hypotension is a common AE. Indeed, we found a statistically significant effect on MAP and heart rate over time, and Mohammed et al reported five cases of unspecified hypotension (26). However, it is important to stress that we did not find any clinically significant change in MAP or heart rate. In our opinion, it is unclear whether the statistically significant changes in MAP and heart rate resulted from 1) a sympatholytic effect of ziconotide, 2) pre-injection anxiety subsiding over time, or 3) an unspecific physiological effect due to the fact that patients remained in the supine position during the first hour postinjection. Overall, the doses used in this and other bolus studies (26-29) were well tolerated, but ziconotide bolus trialing should still only be effectuated in institutions with full-scale resuscitation competence (38).

The median half-life of ziconotide in CSF is 4.5 hours (37). Hence, after 6 hours, CSF levels have dropped significantly. However, the observable pharmacologic effect during infusion treatment develops slowly (i.e., there is a lag between pharmacokinetics and pharmacodynamics) (18) and, as mentioned above, the tissue penetration is slow. Therefore, there is a theoretical possibility that our patients could have experienced late-onset pain reduction (i.e., during the period 6-24 hours post-injection). But the Mohammed study also has a time frame of 6 hours, and they report a proportion of responders of $55 \%$ during that time frame. Crucially, our patients did not report any significant pain reduction for the 24 hours-period post-injection compared to the 24 hours-period pre-injection. Hence, our patients did not seem to experience late-onset pain reduction.

The Polyanalgesic Consensus Conference (PACC) recommendations on trialing for intrathecal analgesia suggest that a patient can be discharged from hospital 12 hours after a single-shot ziconotide trial (provided normal neurologic function) (39). In the light of our own experience, we think that this PACC recommendation is sensible. However, if the patient lives alone or very far away from the hospital, an overnight stay is appropriate, and we do not think the patient should drive for at least 24 hours. On the other hand, if a patient has tolerated a first ziconotide bolus injection without any major side effects, we 
think it is defensible to consider discharging him/her 6 hours after a renewed bolus injection. Of course, thoroughly informing the patient about possible upcoming side effects is especially important before such an early discharge.

Mohammed et al did not report concomitant and previous analgesics. Another difference between our studies is that the cohorts were drawn from different populations. The patients in the present study had post-traumatic and/or post-operative, peripheral neuropathic pain; Mohammed et al had a more heterogeneous sample with about half the patients having unspecified neuropathic pain. Whether this accounts for part of the difference in results between our two studies is unclear, but it is interesting to notice that a recent systematic review has shown that the rate of placebo responders depends on the type of neuropathic pain - for instance, patients with posttraumatic/postsurgical pain seem less likely to be placebo responders than patients with diabetic neuropathic/polyneuropathic pain (40). Might the difference in study populations indicate that our patients were perhaps less prone to placebo than patients in the Mohammed study?

The placebo effect is a well-known phenomenon, not least in pain medicine (41). This phenomenon is highly variable and depends on several contextual factors (42). Interestingly, the placebo effect is thought to be stronger for invasive procedures (43) and for open-label treatments (as opposed to double-blind trials) (42). Still, this invasive and open-label study only had 13\% responders. In another study of this cohort of patients (44), we have recently shown that the CSF levels of beta-endorphin are low compared to healthy controls, perhaps indicating a defective top-down modulation of nociception. In this context, it is interesting to note that the placebo effect, at least in part, is thought to involve top-down modulation of nociception (41). Whether this has any bearing on our results remains an open question.

Far from being placebo responders, 8 out of 16 patients in the present study responded neither to test-SCS nor to ziconotide bolus injections. In the chronic pain setting, this kind of unresponsiveness to available treatment modalities is not unusual, and the concept of "global non-responders" is illustrative in this respect (45). Arguably, our study population consists of a rather high proportion of "global nonresponders". The existence of such "global non-responders" implies a call for more translational pain research, focusing on the mechanisms of different pain conditions in humans. Hence, human biomarker studies are warranted in order to better understand the mechanisms of different neuropathic pain states and why some patients respond to a specific treatment while others don't. 


\section{Conclusion}

We conclude that 1) ziconotide bolus injection trialing seems feasible; 2) the proportion of responders in the present study was low, but there was a subgroup of responders; 3) AEs were as expected according to the SPC, and no SAE occurred; 4) the predictive power of ziconotide bolus trialing remains unclear; 5) the pharmacological profile of ziconotide (with very slow tissue penetration due to high hydrophilicity) calls the rationale for bolus trialing into question; 6) patients refractory to all available treatment modalities are a reminder of the need for more research into the mechanisms of different pain conditions. 


\section{Acknowledgments}

The study was supported by grants from County Council of Östergötland and the Swedish Research Council. There are no conflicts of interest.

Thanks to study nurses Marie Berggården and Anna Petersson for excellent practical assistance when the patients visited the Pain and Rehabilitation Centre. Thanks also to statistician Karl Wahlin. 


\section{References}

1. Onofrio BM, Yaksh TL, Arnold PG. Continuous low-dose intrathecal morphine administration in the treatment of chronic pain of malignant origin. Mayo Clin Proc. 1981;56:516-520.

2. Portenoy RK. Treatment of cancer pain. Lancet. 2011;377:2236-2247.

3. Backryd E, Larsson B. Movement-evoked breakthrough cancer pain despite intrathecal analgesia: a prospective series. Acta Anaesthesiol Scand. 2011;55:11391146.

4. Myers J, Chan V, Jarvis V, Walker-Dilks C. Intraspinal techniques for pain management in cancer patients: a systematic review. Support Care Cancer. 2010;18:137-149.

5. Turk DC, Wilson HD, Cahana A. Treatment of chronic non-cancer pain. Lancet. 2011;377:2226-2235.

6. Turner JA, Sears JM, Loeser JD. Programmable intrathecal opioid delivery systems for chronic noncancer pain: a systematic review of effectiveness and complications. Clin J Pain. 2007;23:180-195.

7. Hayek SM, Deer TR, Pope JE, Panchal SJ, Patel VB. Intrathecal therapy for cancer and non-cancer pain. Pain Physician. 2011;14:219-248.

8. $\quad$ Erdine S, De Andres J. Drug delivery systems. Pain Pract. 2006;6:51-57.

9. Kress HG, Simpson KH, Marchettini P, Ver Donck A, Varrassi G. Intrathecal therapy: what has changed with the introduction of ziconotide. Pain Pract. 2009;9:338-347.

10. Miljanich GP. Ziconotide: neuronal calcium channel blocker for treating severe chronic pain. Curr Med Chem. 2004;11:3029-3040.

11. Pope JE, Deer TR. Ziconotide: a clinical update and pharmacologic review. Expert Opin Pharmacother. 2013;14:957-966.

12. Staats PS, Yearwood $T$, Charapata SG, et al. Intrathecal ziconotide in the treatment of refractory pain in patients with cancer or AIDS: a randomized controlled trial. JAMA. 2004;291:63-70.

13. Rauck RL, Wallace MS, Leong MS, et al. A randomized, double-blind, placebocontrolled study of intrathecal ziconotide in adults with severe chronic pain. J Pain Symptom Manage. 2006;31:393-406. 
14. Wallace M, Charapata S, Fisher R, et al. Intrathecal ziconotide in the treatment of chronic nonmalignant pain: a randomized, double-blind, placebo-controlled clinical trial. Neuromodulation. 2006;9:75-86.

15. Deer TR, Prager J, Levy R, et al. Polyanalgesic Consensus Conference 2012: recommendations for the management of pain by intrathecal (intraspinal) drug delivery: report of an interdisciplinary expert panel. Neuromodulation. 2012;15:436464; discussion 464-436.

16. Deer T, Krames E, Hassenbusch S, et al. Polyanalgesic Consensus Conference 2007: recommendations for the management of pain by intrathecal (intraspinal) drug delivery: report of an interdisciplinary expert panel. Neuromodulation. 2007;10:300328.

17. Schmidtko A, Lotsch J, Freynhagen R, Geisslinger G. Ziconotide for treatment of severe chronic pain. Lancet. 2010;375:1569-1577.

18. Smith HS, Deer TR. Safety and efficacy of intrathecal ziconotide in the management of severe chronic pain. Ther Clin Risk Manag. 2009;5:521-534.

19. Sanford M. Intrathecal Ziconotide: A Review of Its Use in Patients with Chronic Pain Refractory to Other Systemic or Intrathecal Analgesics. CNS Drugs. 2013;27:9891002.

20. Obafemi A, Roth B. Prolonged delirium with psychotic features from omega conotoxin toxicity. Pain Med. 2013;14:447-448.

21. Maier C, Gockel HH, Gruhn K, Krumova EK, Edel MA. Increased risk of suicide under intrathecal ziconotide treatment? - a warning. Pain. 2011;152:235-237.

22. Wallace MS, Rauck R, Fisher R, Charapata SG, Ellis D, Dissanayake S. Intrathecal ziconotide for severe chronic pain: safety and tolerability results of an open-label, long-term trial. Anesth Analg. 2008;106:628-637.

23. Burton AW, Deer TR, Wallace MS, Rauck RL, Grigsby E. Considerations and methodology for trialing ziconotide. Pain Physician. 2010;13:23-33.

24. Ahmed S, Martin N, Chang Y. Patient selection and trial methods for intraspinal drug delivery for chronic pain: a national survey. Neuromodulation. 2005;8:112-120.

25. Anderson V, Burchiel K, Cooke B. A prospective, randomized trial of intrathecal injection vs. epidural infusion in the selection of patients for continuous intrathecal opioid therapy. Neuromodulation. 2003;6:142-152.

26. Mohammed SI, Eldabe S, Simpson KH, et al. Bolus intrathecal injection of ziconotide $(\operatorname{Prialt}(\mathrm{R}))$ to evaluate the option of continuous administration via an implanted 
intrathecal drug delivery (ITDD) system: a pilot study. Neuromodulation. 2013;16:576-581; discussion 582.

27. Grigsby E, McGlothlen G. Single-shot Bolus Trialing to Assess Patient Response to Intrathecal Ziconotide. http://asra.com/display_fall_2010.php?id=14. 2010; retrieved 7 december 2012.

28. Okano D. Single-Shot Intrathecal Ziconotide to Predict Its Pump Infusion Effect. http://aapm.confex.com/aapm/2008am/techprogram/P2851.HTM. 2008; retrieved 7 december 2012.

29. Rosenblum S. Intrathecal Bolus Injection of Ziconotide for Severe Chronic Pain: Evaluation of Analgesic Response. Anesthesiology. 2008;109:A1566.

30. Treede RD, Jensen TS, Campbell JN, et al. Neuropathic pain: redefinition and a grading system for clinical and research purposes. Neurology. 2008;70:1630-1635.

31. Hawker GA, Mian S, Kendzerska T, French M. Measures of adult pain: Visual Analog Scale for Pain (VAS Pain), Numeric Rating Scale for Pain (NRS Pain), McGill Pain Questionnaire (MPQ), Short-Form McGill Pain Questionnaire (SFMPQ), Chronic Pain Grade Scale (CPGS), Short Form-36 Bodily Pain Scale (SF-36 BPS), and Measure of Intermittent and Constant Osteoarthritis Pain (ICOAP). Arthritis Care Res (Hoboken). 2011;63 Suppl 11:S240-252.

32. Dworkin RH, Turk DC, Farrar JT, et al. Core outcome measures for chronic pain clinical trials: IMMPACT recommendations. Pain. 2005;113:9-19.

33. Ward M, Langton JA. Blood pressure measurement. Contin Educ Anaesth Crit Care Pain 2007;7:122-126.

34. Matthews JN, Altman DG, Campbell MJ, Royston P. Analysis of serial measurements in medical research. BMJ. 1990;300:230-235.

35. Arendt K, Demaerschalk BM, Wingerchuk DM, Camann W. Atraumatic lumbar puncture needles: after all these years, are we still missing the point? Neurologist. 2009;15:17-20.

36. Yaksh TL, de Kater A, Dean R, Best BM, Miljanich GP. Pharmacokinetic analysis of ziconotide (SNX-111), an intrathecal N-type calcium channel blocking analgesic, delivered by bolus and infusion in the dog. Neuromodulation. 2012;15:508-519; discussion 519.

37. Wermeling D, Drass M, Ellis D, et al. Pharmacokinetics and pharmacodynamics of intrathecal ziconotide in chronic pain patients. J Clin Pharmacol. 2003;43:624-636. 
38. Heifets BD, Smith SM, Leong MS. Acute Cardiovascular Toxicity of Low-Dose Intrathecal Ziconotide. Pain Med. 2013.

39. Deer TR, Prager J, Levy R, et al. Polyanalgesic Consensus Conference--2012: recommendations on trialing for intrathecal (intraspinal) drug delivery: report of an interdisciplinary expert panel. Neuromodulation. 2012;15:420-435; discussion 435 .

40. Cepeda MS, Berlin JA, Gao CY, Wiegand F, Wada DR. Placebo response changes depending on the neuropathic pain syndrome: results of a systematic review and metaanalysis. Pain Med. 2012;13:575-595.

41. Colloca L, Klinger R, Flor H, Bingel U. Placebo analgesia: psychological and neurobiological mechanisms. Pain. 2013;154:511-514.

42. Manchikanti L, Giordano J, Fellows B, Hirsch JA. Placebo and nocebo in interventional pain management: a friend or a foe--or simply foes? Pain Physician. 2011;14:E157-175.

43. Klinger R, Colloca L, Bingel U, Flor H. Placebo analgesia: clinical applications. Pain. 2014;155:1055-1058.

44. Backryd E, Ghafouri B, Larsson B, Gerdle B. Do low levels of Beta-endorphin in the cerebrospinal fluid indicate defective top-down inhibition in patients with chronic neuropathic pain? A cross-sectional, comparative study. Pain Med. 2014;15:111-119.

45. Lemming D, Sorensen J, Graven-Nielsen T, Arendt-Nielsen L, Gerdle B. The responses to pharmacological challenges and experimental pain in patients with chronic whiplash-associated pain. Clin J Pain. 2005;21:412-421. 


\section{Legends to the figures}

\section{Figure 1}

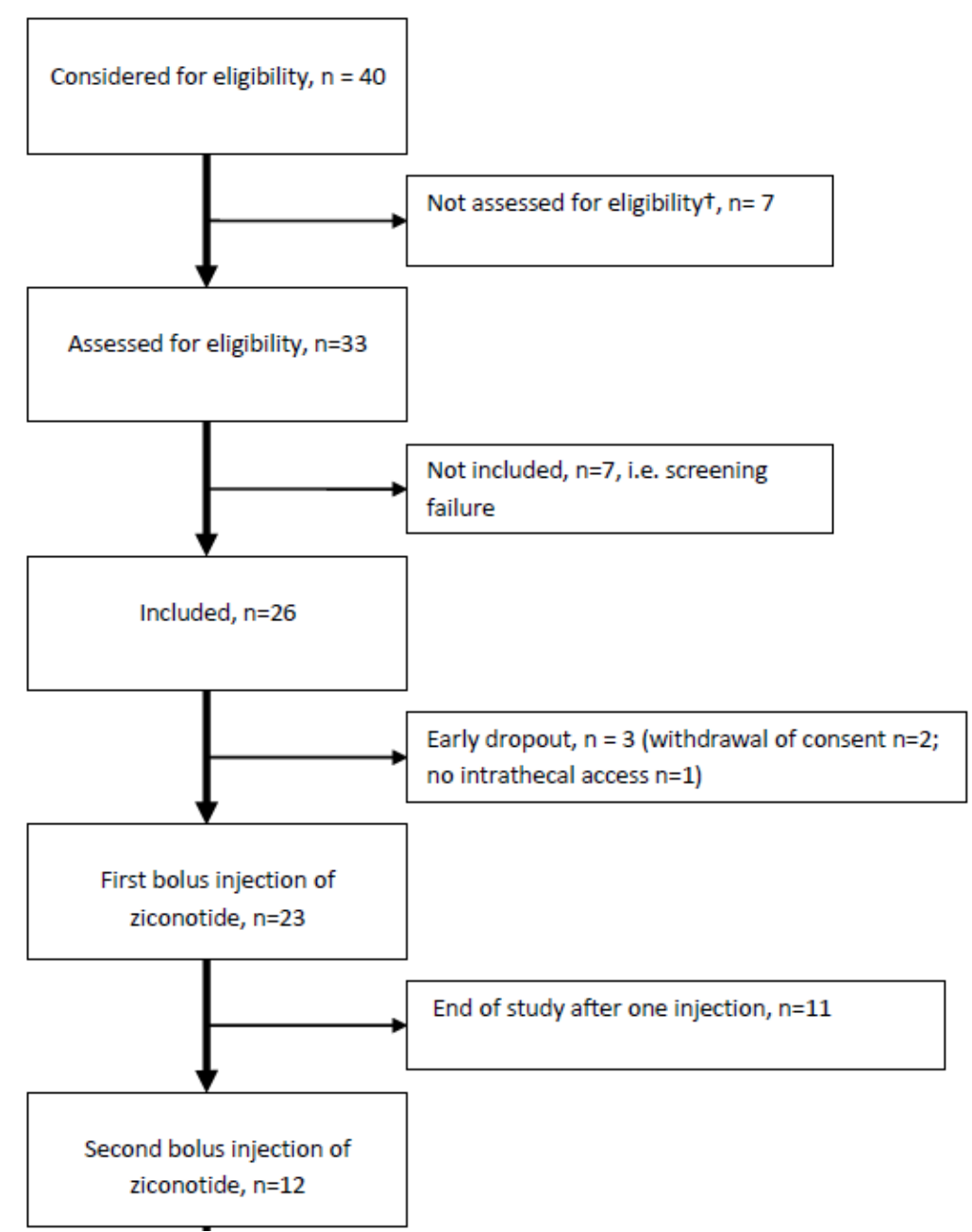

\section{Figure 1: Patient flow diagram.}

$\dagger=$ Patients who did not want to be assessed, or who beforehand unmistakably could be said to fulfill an exclusion criterion. 


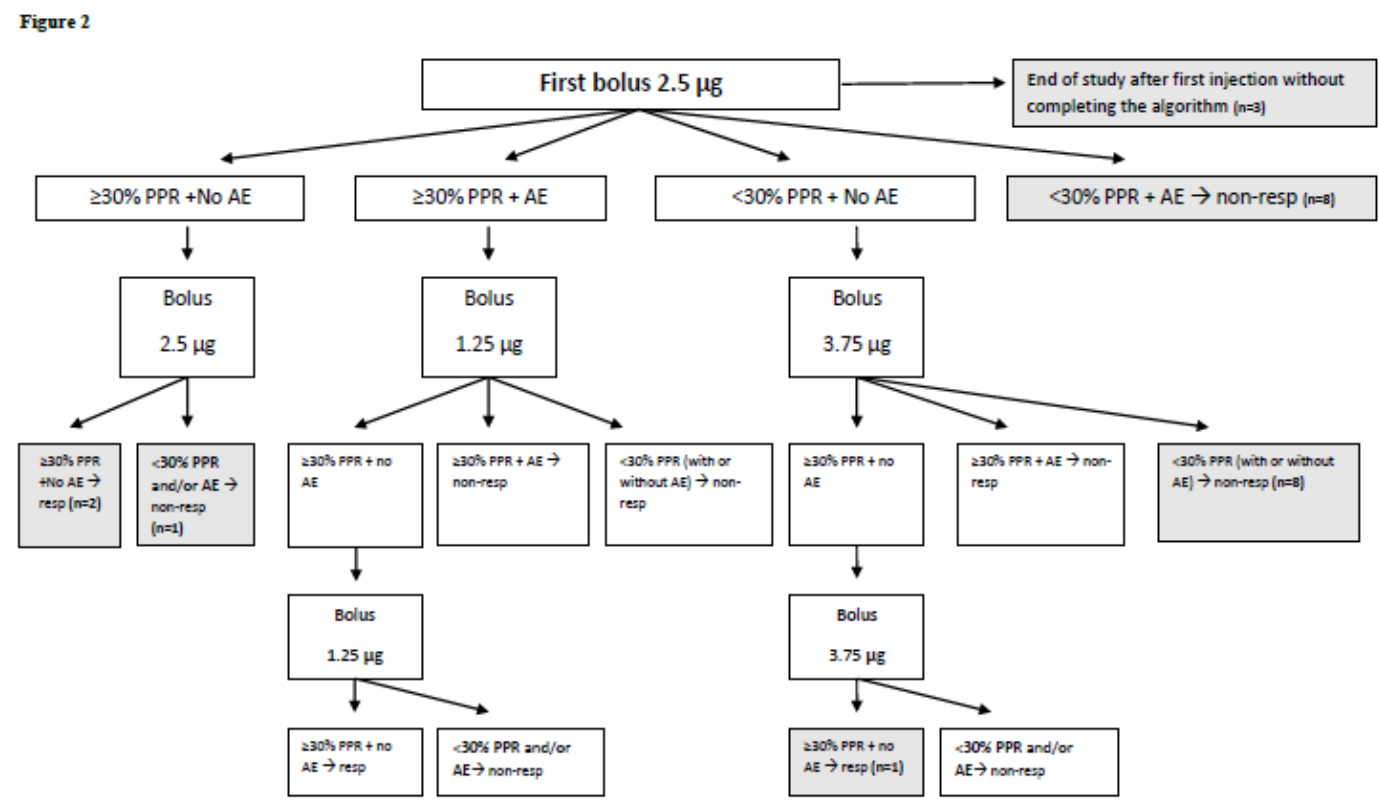

Figure 2: Intrathecal ziconotide bolus injection flow chart. Patients $(n=23)$ progressed in the algorithm according to how they responded to each bolus injection. Shaded boxes represent where patients finally ended up in the algorithm. $\mathrm{AE}=$ significant adverse event according to study definition, see text. PPR= Percentage Pain Reduction. Resp = responder to ziconotide (i.e. positive outcome). Non-resp= nonresponder to ziconotide (i.e. negative outcome). 


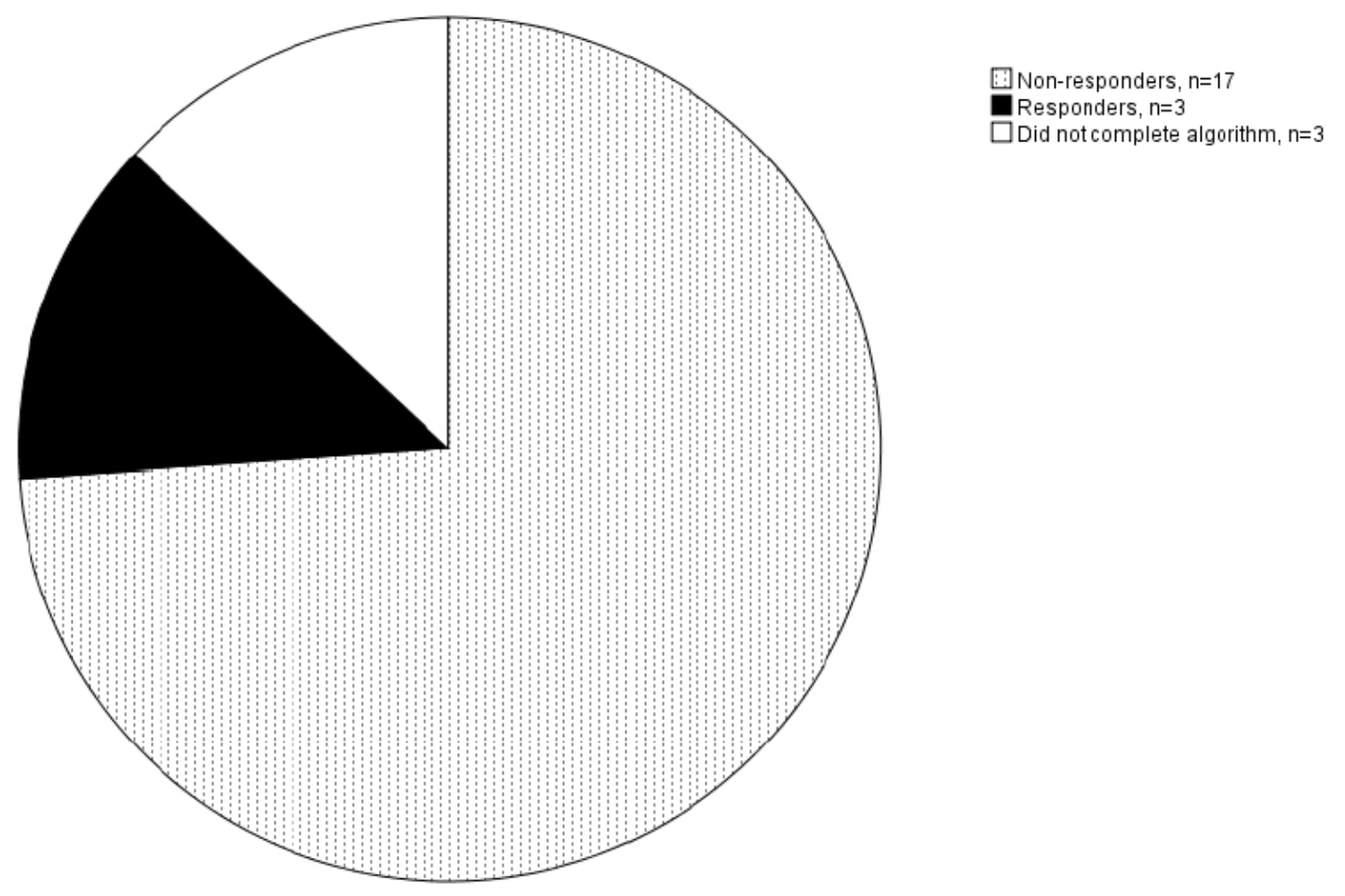

Figure 3: Proportion of responders and non-responders to ziconotide bolus injection trialing according to study algorithm. Three patients did not complete the study algorithm. 


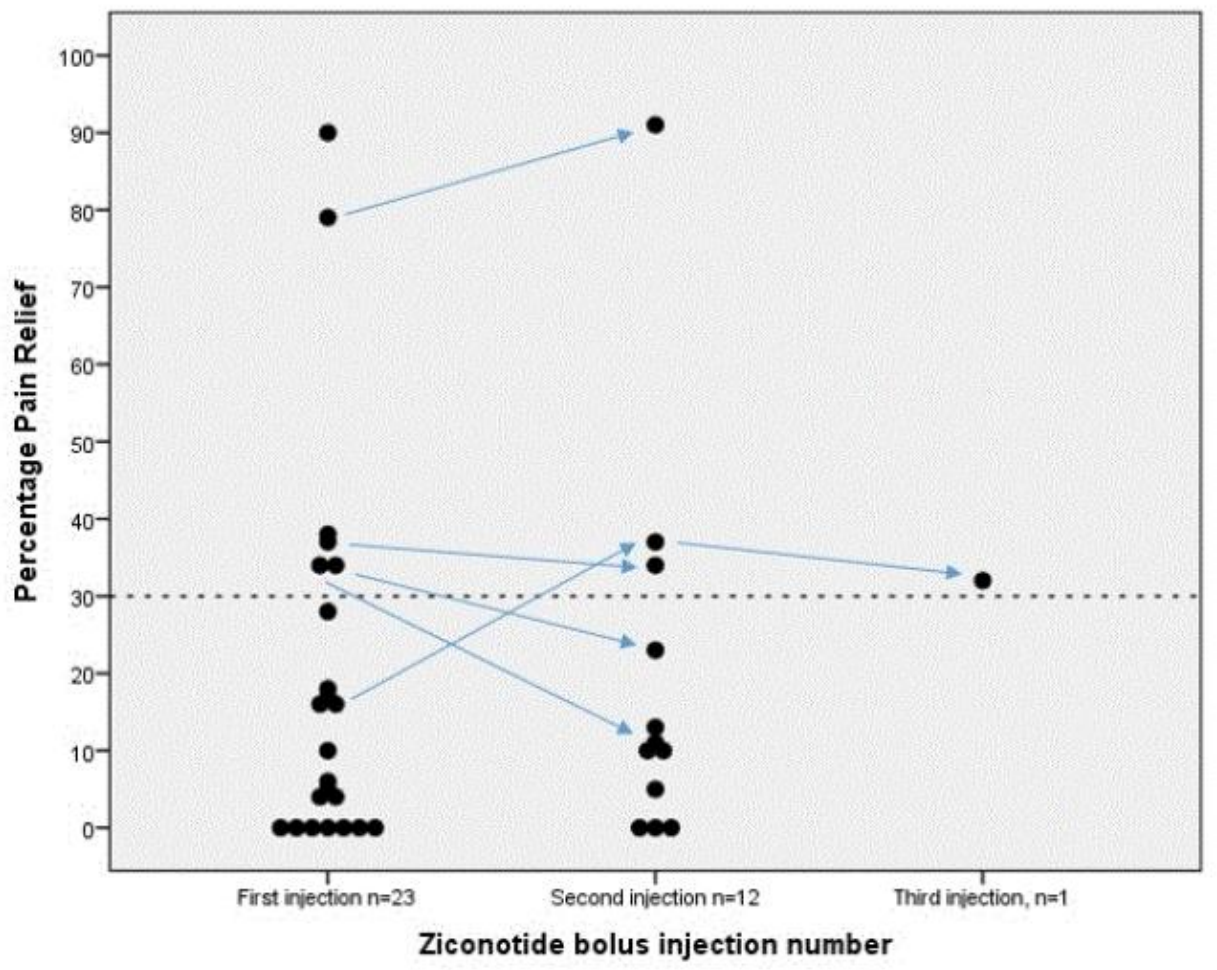

Figure 4: Percentage Pain Reduction of each bolus injection. Each dot represents one injection of ziconotide, and the dotted line represents 30\% pain relief. Negative values are depicted as zero. The first injection dose of ziconotide was always $2.5 \mu \mathrm{g}$. The second and third injection doses varied according to the study algorithm $(1.25,2.5$, or $3.75 \mu \mathrm{g})$. Only one patient completed three injections. Arrows indicate how patients experiencing more than $30 \%$ pain relief on at least one occasion and receiving more than one injection progressed in the study. 


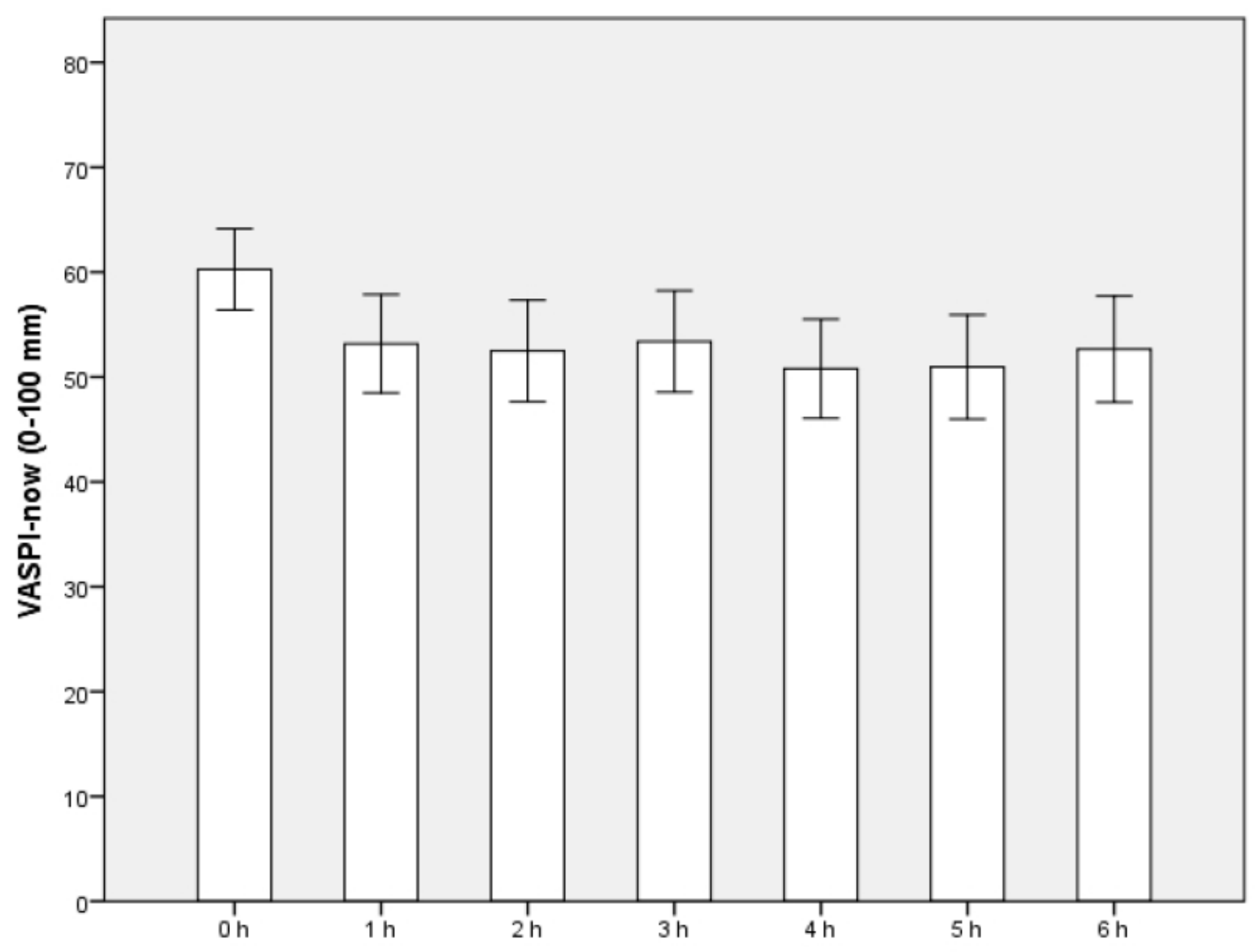

Figure 5: Pain intensity 0-6 hours post-injection $(\mathbf{n = 2 3})$. Timepoint $0 \mathrm{~h}$ is immediately pre-injection. Pain intensity assessed as "Visual Analogue Scale Pain Intensity now" (VASPI-now) 0-100 mm. A summary measures approach was used, see text. Data are presented as mean \pm SEM. RM-ANOVA showed significant changes over time $(\mathrm{p}=0.047)$ after a mean ziconotide dose of $2.75 \mu \mathrm{g}$. Pairwise post hoc analysis showed that timepoint $0 \mathrm{~h}$ differed significantly from timepoints $1 \mathrm{~h}(\mathrm{p}=0.022), 2 \mathrm{~h}(\mathrm{p}=0.018), 4 \mathrm{~h}$ ( $\mathrm{p}=0.017)$, and $5 \mathrm{~h}(\mathrm{p}=0.016)$. 


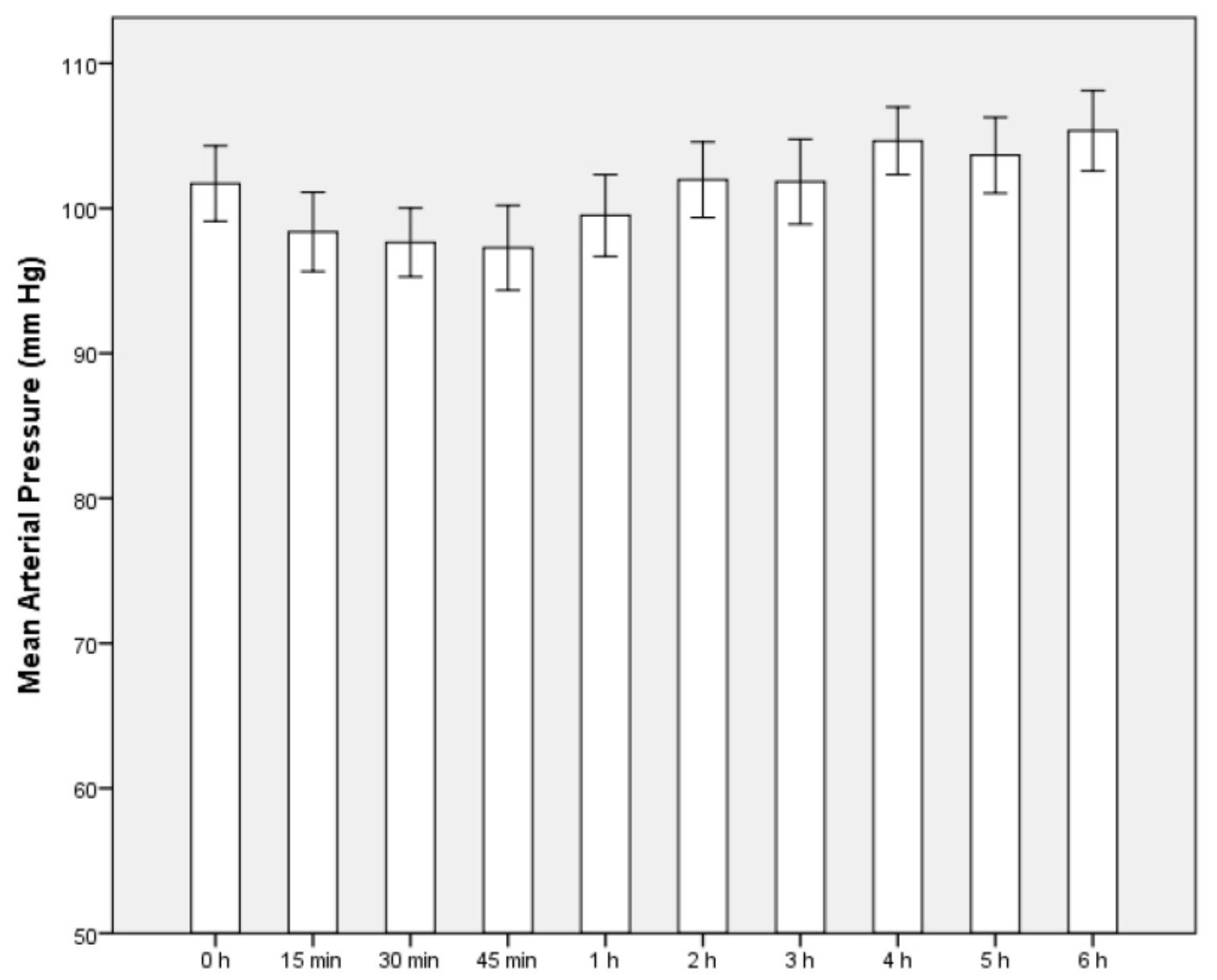

Figure 6: Cardiovascular safety 0-6 hours post-injection $(\mathbf{n = 2 3})$. Time point 0 is immediately preinjection. A summary measures approach was used, see text. Data are presented as mean \pm SEM. RMANOVA showed statistically significant changes of both MAP $(p<0.001)$ and heart rate $(p<0.001)$ over time, see text.

a. Mean Arterial Pressure (mm Hg)

b. Heart Rate (beats per minute) 


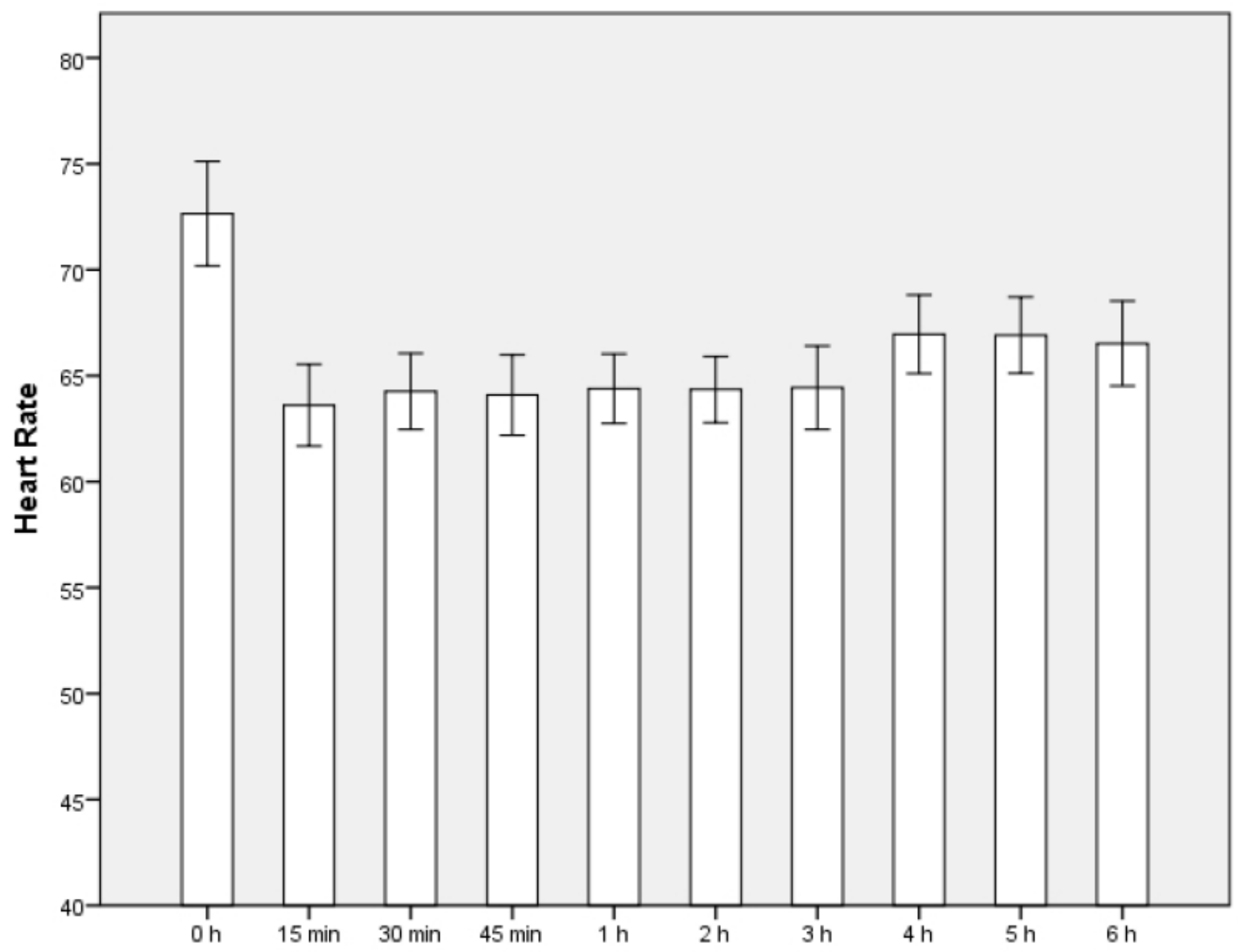

Figure 6: Cardiovascular safety 0-6 hours post-injection $(\mathbf{n = 2 3})$. Time point 0 is immediately preinjection. A summary measures approach was used, see text. Data are presented as mean \pm SEM. RMANOVA showed statistically significant changes of both MAP $(p<0.001)$ and heart rate $(p<0.001)$ over time, see text.

c. Mean Arterial Pressure $(\mathrm{mm} \mathrm{Hg})$

d. Heart Rate (beats per minute) 
Table 1: Clinical characteristics of injected pain patients $(n=23)$

\begin{tabular}{|c|c|c|c|c|c|c|c|c|c|}
\hline $\begin{array}{l}\text { Patient } \\
\text { study } \\
\text { id }\end{array}$ & $\begin{array}{l}\text { ICD-10 } \\
\text { diagn }^{1}\end{array}$ & $\begin{array}{l}\text { Pain } \\
\text { duration } \\
\text { months }\end{array}$ & $\begin{array}{l}\text { Pain } \\
\text { Intensity }^{2}\end{array}$ & $\begin{array}{l}\text { Concomitant } \\
\text { analgesics }^{3}\end{array}$ & $\begin{array}{l}\text { Con. } \\
\text { opioid } \\
\text { dose }^{4}\end{array}$ & Co-morbidities ${ }^{5}$ & $\begin{array}{l}\text { Previous } \\
\text { analgesics }^{6}\end{array}$ & $\begin{array}{l}\text { SCS } \\
\text { response }^{7}\end{array}$ & $\begin{array}{l}\text { Study } \\
\text { outcome }^{8}\end{array}$ \\
\hline 001 & S74.1 & 30 & 82 & $P$ & 0 & & $\mathrm{AD}, \mathrm{Gab}$ & Non-resp & Non-resp \\
\hline 002 & S34.2 & 120 & 75 & & 0 & psoriasis; tension-type headache & AD, Gab, Op & Non-resp & Non-resp \\
\hline 003 & S34.2 & 39 & 72 & & 0 & HT; PMR & $A D, G a b, O p$ & Non-resp & Non-resp \\
\hline 005 & S74.0 & 120 & 82 & $P$ & 0 & postural hypotension; PVD; VC & $A D$, Gab, Op & Non-resp & Non-resp \\
\hline 006 & S54.9 & 300 & 64 & $\mathrm{P}, \mathrm{Op}$ & 30 & & $A D, G a b$ & Non-resp & Resp \\
\hline 008 & S54.0 & 65 & 42 & AD, Gab & 0 & & Op & Resp & Non-resp \\
\hline 009 & S94.9 & 58 & 60 & $\mathrm{P}, \mathrm{Op}$ & 150 & & $\mathrm{AD}, \mathrm{Gab}$ & Non-resp & Resp \\
\hline 010 & S34.2 & 72 & 72 & Op & 50 & HT; psoriasis & Gab & Resp & Resp \\
\hline 011 & S34.2 & 60 & 59 & $P, N S A I D, A D$, Gab, Op & 180 & angina; OLD & & NA & Non-resp \\
\hline 012 & S34.2 & 36 & 87 & $\mathrm{P}$ & 0 & anemia; dyspepsia; HT & $A D$, Gab, Op & Non-resp & Non-resp \\
\hline 013 & S34.2 & 120 & 40 & & 0 & & $A D, O p$ & Non-resp & NA \\
\hline 014 & S34.3 & 79 & 78 & $P$, NSAID, AD, Gab, Op & 480 & & & NA & Non-resp \\
\hline 015 & S34.2 & 180 & 71 & $A D, G a b, O p$ & 32 & AN; diabetes; angina; PAD; dyspepsia & & Resp & NA \\
\hline 016 & G62.9 & 78 & 68 & & 0 & none & $A D, G a b, O p$ & Resp & Non-resp \\
\hline 017 & S14.2 & 12 & 58 & $P, A D, G a b$ & 0 & fibromyalgia & & NA & Non-resp \\
\hline 018 & S34.2 & 48 & 83 & P, Gab, Op & 50 & localized bladder tumor & $A D$ & Resp & Non-resp \\
\hline 019 & S14.2 & 18 & 58 & $A D, G a b, O p$ & 30 & & & Non-resp & Non-resp \\
\hline 021 & S34.2 & 120 & 74 & $P, O p$ & 20 & depression & AD, Gab & Resp & Non-resp \\
\hline 022 & S14.2 & 18 & 84 & & 0 & $\mathrm{HT}$ & $A D, G a b, O p$ & NA & Non-resp \\
\hline 023 & S34.2 & 84 & 90 & Gab, Op & 225 & & $A D$ & Resp & Non-resp \\
\hline 024 & S34.6 & 21 & 75 & Gab & 0 & & $A D, G a b, O p$ & NA & NA \\
\hline 025 & S84.2 & 96 & 76 & Op & 240 & HT; diabetes; hyperlipidemia & $\mathrm{AD}, \mathrm{Gab}$ & Non-resp & Non-resp \\
\hline 026 & S84.1 & 52 & 94 & $A D, G a b, O p$ & 260 & & & Non-resp & Non-resp \\
\hline
\end{tabular}


1. International Classification of Diseases (ICD-10) key: S14.2 - injury of nerve root of cervical spine; S34.2 - injury of nerve root of lumbar and sacral spine (i.e. failed back surgery syndrome with radiculopathy); S34.3 - injury of cauda equina; S34.6- injury of peripheral nerves of abdomen; S54.0 - injury of ulnar nerve at forearm level; S54.9 - injury of unspecified nerve at forearm level; S74.0 - injury of sciatic nerve at hip and thigh level; S74.1 - injury of femoral nerve at hip and thigh level; S84.1- injury of peroneal nerve at lower leg level; S84.2 - injury of cutaneous sensory nerve at lower leg level; S94.9 - injury of unspecified nerve at ankle and foot level; G62.9 - polyneuropathy.

2. Visual Analogue Scale Pain Intensity during week before inclusion (VASPI-inclusion), 0-100 mm.

3. Abbreviations for analgesics: $\mathrm{P}=$ paracetamol; NSAID=Non-steroidal anti-inflammatory drug; $\mathrm{AD}=$ amitriptyline or duloxetine; $\mathrm{Gab}=$ gabapentinoids, i.e. gabapentin or pregabalin; $\mathrm{Op}=$ opioids.

4. Concomitant dose in oral morphine equivalents, mg.

5. Abbreviations: $\mathrm{AN}=$ autonomic neuropathy; $\mathrm{HT}=$ hypertension; $\mathrm{OLD}=$ obstructive lung disease (mild); $\mathrm{PAD}=$ panic anxiety disorder; PMR=Polymyalgia rheumatica; $\mathrm{PVD}=$ posterior vitreous detachment; $\mathrm{VC}=$ vertebral compressions (no longer painful). One of the patients also had a history of alcohol dependency.

6. Paracetamol and NSAID not included. For abbreviations, see note 3 above.

7. Resp=Responder to Spinal Cord Stimulation (SCS); Non-resp=Non-responder to SCS; NA=Not applicable, i.e. did not try SCS.

8. Resp=Responder to ziconotide bolus injections (according to study algorithm, see text); Non-resp=Non-responder to ziconotide; NA= Not applicable, i.e did not complete the study algorithm 
